\title{
2 Was erwarten Wissenschaftler von zentralisierten Biobanken? Eine qualitative Stakeholder-Analyse
}

Antje Schütt, Wiebke Lesch und Roland Jahns

- Neben Probenspendern und Öffentlichkeit gelangen zunehmend auch andere Stakeholder in den Fokus einer professionellen Kommunikation von Biobanken.

- Eine strukturierte Stakeholder-Analyse hilft, die für Biobanken relevanten Stakeholder zu identifizieren, ihre Interessen und ihren Einfluss zu beschreiben, sie zu priorisieren und geeignete Interaktionsstrategien abzuleiten.

- Die Weiterentwicklung von biomedizinischen Forschungsinfrastrukturen führt zu einer klareren Aufgabenverteilung zwischen den Biobankakteuren und damit zu einer deutlicheren Trennung der unterschiedlichen Rollen. Dies bringt erhöhte Kommunikationsanforderungen mit sich.

- Biobanken müssen im Klinikum als Infrastruktureinrichtung bekannt werden und Vertrauen zu den internen Stakeholdergruppen aufbauen.

- Der German Biobank Node wird allen Biobanken Materialien und Unterstützungsangebote für Stakeholderkommunikation und -engagement zur Verfügung stellen. 


\subsection{Biobanken haben Interaktionen auf vielen Ebenen}

Biobanken sind wichtige, auf Langfristigkeit angelegte Forschungsinfrastrukturen, die die Grundlage für die Entwicklung der Präzisionsmedizin (personalisierten/stratifizierten Medizin) bilden. Sie sind weltweit noch junge Einrichtungen, die nicht nur in der Öffentlichkeit (European Commission 2012), sondern auch unter Wissenschaftlern und Klinikern häufig noch unbekannt sind, und deren Fortbestand von der Unterstützung durch verschiedene Communities abhängt (Cañada et al. 2015). Ein gutes Management der Interaktion mit den relevanten Bezugsgruppen auf Basis einer StakeholderAnalyse ist deshalb eine wichtige Aufgabe im Projektmanagement einer Biobanken-Infrastruktur (Bjugn u. Casati 2012).

In der wissenschaftlichen Literatur - vor allem aus dem anglo-amerikanischen und nordeuropäischen Raum - lag der Hauptfokus beim Thema Biobanken-Kommunikation und -Partizipation bisher auf der Information und Einbeziehung von Patienten und Probanden bzw. Bürgern oder „der Öffentlichkeit“. Das Spektrum der Aktivitäten und Zielsetzungen reicht hier von der Steigerung der Bekanntheit von Biobanken als Forschungsressource mit dem Ziel, sie gesellschaftlich zu legitimieren und die Bereitschaft zur Teilnahme zu verbessern (Gaskell u. Gottweis 2011; Lesch et al. 2016), über die Erhebung der Einstellungen, Sichtweisen und Bedenken von Probenspendern und Öffentlichkeit, um die Argumentation und die Angebote daraufhin anpassen zu können (Lesch et al. 2016; Mitchell et al. 2015), bis hin zur Integration von Patienten in die Steuerungsgremien oder gar der Etablierung von Biobanken durch die Patienten selbst (Cañada et al. 2015; Mitchell et al. 2015).

Die Bedeutung anderer Stakeholder - der medizinischen Institutionen und der Förderorganisationen als wichtigste Geldgeber oder der Forscher als die eigentlichen „Kunden“ einer Biobank (Simeon-Dubach u. Watson 2014) wird erst in letzter Zeit stärker in den Vordergrund gestellt und systematisch erforscht. Auch die in der Krankenversorgung tätigen Berufsgruppen, die im Behandlungskontext Proben entnehmen (sollen), die dann für Forschungszwecke in einer zentralen Biobank gelagert werden, rücken zunehmend in den Fokus, denn ohne ihren Beitrag sind die Möglichkeiten der Probengewinnung sehr limitiert (Caixeiro et al. 2015; Gottweis u. Lauss 2012). Cañada et al. argumentieren sogar, dass die direkte Interaktion mit der Öffentlichkeit nur einen geringen Anteil der Interaktion mit Stakeholdern ausmacht und dass die Einbeziehung anderer Gruppen - Krankenhäuser und Kliniker, andere Biobanken, öffentliche Verwaltungen und Förderer, Industrie sowie Forscher - möglicherweise noch wichtiger ist, wenn die Biobanken wirklich wissenschaftlichen Mehrwert generieren wollen (Cañada et al. 2015).

Für das Kommunikations-Teilprojekt im German Biobank Node (GBN) sollten für die Aufbauphase zentralisierter Biobanken an Universitätskliniken neben Patienten und Probanden als Probenspendern - zunächst explorativ 
2 Was erwarten Wissenschaftler von zentralisierten Biobanken?

die Erwartungen und Einstellungen von Wissenschaftlern und Klinikern gegenüber den neuen Infrastrukturen sowie die Kommunikationsanforderungen an die Biobankverantwortlichen eruiert werden, um daraus eine entsprechende Kommunikations- und Engagementstrategie zu entwickeln und ein Unterstützungsangebot für die Biobanken abzuleiten.

\section{Eine Stakeholder-Analyse durchführen}

Als Stakeholder werden die Bezugs- oder Anspruchsgruppen einer Organisation (oder eines Politikbereiches bzw. Themas) bezeichnet, die von dieser beeinflusst werden und diese ihrerseits beeinflussen (Bjugn u. Casati 2012; Buse et al. 2012). Nur auf Basis einer Identifizierung, Charakterisierung und Priorisierung dieser Gruppen ist es möglich, das Verhältnis zu und die Interaktion mit ihnen strategisch zu planen. Es sind zahlreiche Methoden der StakeholderAnalyse beschrieben worden, im Grundsatz gehen sie aber in vergleichbarer Weise vor (Buse et al. 2012).

\section{Identifizierung von Stakeholdern}

Der erste Schritt ist die Identifizierung von relevanten Bezugsgruppen. In der Praxis geschieht dies in der Regel durch ein Brainstorming, in das möglichst Personen eingebunden werden sollten, die das thematische Umfeld gut kennen (Buse et al. 2012). Es empfiehlt sich, die Liste ggf. durch eine Person prüfen zu lassen, die nicht dem Projektteam angehört (Bjugn u. Casati 2012). Stakeholder einer Biobank können als klinikumsintern oder -extern (Bjugn u. Casati 2012) oder nach der Art ihrer Assoziierung mit der Biobank klassifiziert werden (s. Abb. 1): direkt assoziiert (z.B. Mitarbeiter, Probenspender), in der engeren Umgebung und mit direkter Interaktion (z.B. wissenschaftliche Probennutzer) oder im weiteren Umfeld (z.B. andere Biobanken, Behörden, Gesetzgeber).

\section{Beschreibung ihrer Interessen, ihrer Werte und ihres Einflusses}

Sinnvoll wird eine solche Zusammenstellung erst dann, wenn die Stakeholder hinsichtlich ihrer vermuteten oder auf der Basis von Forschungsdaten (qualitativ, z.B. Interviews, quantitativ, z.B. Surveys) beschriebenen Positionierungen und Einstellungen gegenüber dem Thema Biobanken bzw. der konkreten Biobank (z.B. positiv/negativ, fördernd/hemmend, geringes/hohes Interesse etc.) sowie hinsichtlich ihrer Möglichkeiten der Einflussnahme charakterisiert werden können. So lassen sich die Stakeholder beispielsweise in einer EinflussInteressen-Matrix kartieren (s. Abb. 2), wobei ggf. auch andere entscheidende Ordnungsmerkmale für die Kartierung gewählt werden können.

Aus der jeweiligen Platzierung ergeben sich die entsprechenden Interaktionsstrategien. So muss dafür gesorgt werden, dass die Interaktion mit den Personen und Gruppen, die ein hohes Interesse und großen Einfluss haben, sehr eng ist, während es bei denjenigen, die zwar viel Einfluss, aber wenig Interesse haben, darauf ankommt, sie zufrieden zu halten. Bei Stakeholdern, die sehr 


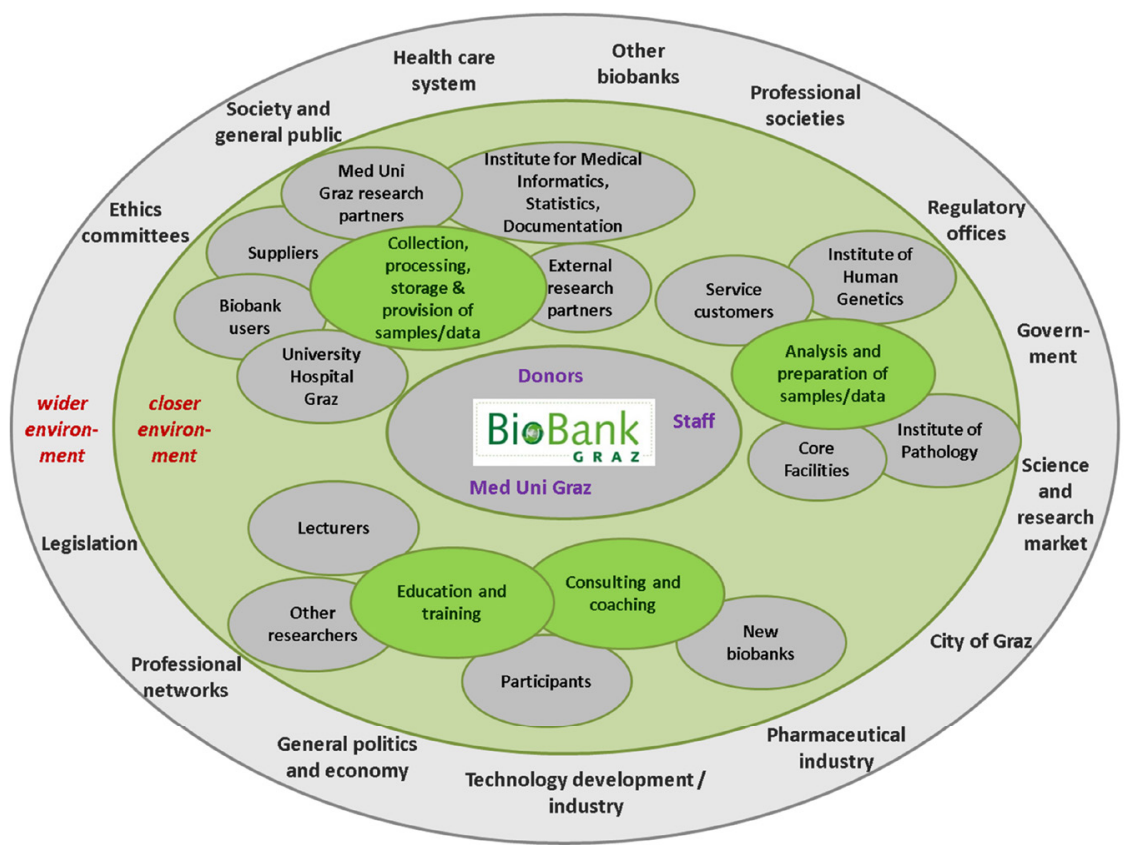

Abb. 1 Beispiel einer Stakeholder-Analyse für eine Biobank (Quelle: Sargsyan K, Story P Granitz G, Macheiner T, Huppertz B: Stakeholder Analysis of an Academic Biobank. Posterpräsentation im Rahmen der Europe Biobank Week 2016, online verfügbar unter http://www.medunigraz.at/fileadmin/forschen/biobank/pdf/2016_EBW_Poster_ Stakeholder.pdf, Abruf: 02.11.2016)

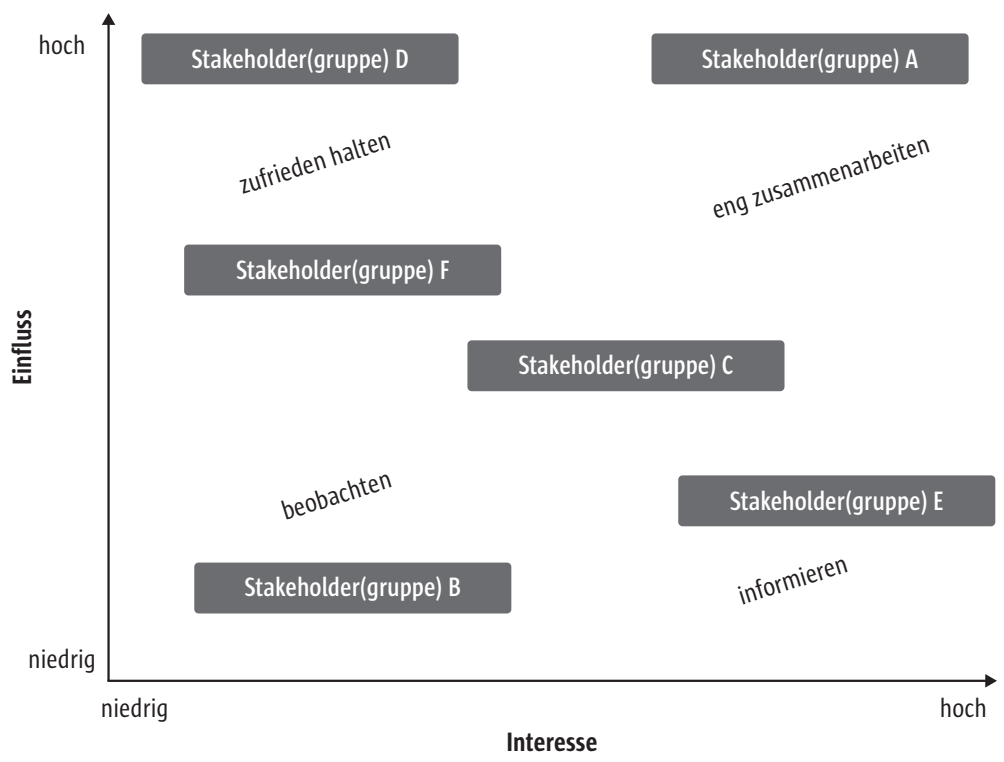

Abb. 2 Muster einer Einfluss-Interessen-Matrix zur Charakterisierung von Stakeholdern 
2 Was erwarten Wissenschaftler von zentralisierten Biobanken?

interessiert sind, aber wenig Einfluss haben, ist es ausreichend, sie regelmäßig mit Informationen zu versorgen, während diejenigen, die weder Interesse noch Einfluss haben, lediglich beobachtet werden sollten, um eventuell kritische Veränderungen frühzeitig zu erkennen.

\section{Priorisierung}

Da Ressourcen in der Regel begrenzt sind, ist es unerlässlich, die Stakeholder auf der Basis der vorhergehenden Charakterisierung zu priorisieren, dabei eventuell verschiedene Gruppen zusammenzufassen, und die strategische Maßnahmenplanung auf ausgewählte Stakeholdergruppen zu beschränken.

\section{Interaktions-Strategie}

Auf dieser Basis wird eine spezifische Interaktions-Strategie (engagement strategy) für jede der priorisierten Stakeholdergruppen entwickelt. Was unter „Engagement " verstanden wird, ist unterschiedlich und mit verschiedenen Stufenmodellen beschrieben worden. Das Spektrum der Stufen reicht von Aktivitäten, die die Erwartungen oder Ansichten der Stakeholder verändern sollen, über Bildungsmaßnahmen und das Einholen von Rat bis hin zur gleichberechtigten Partnerschaft und tatsächlich geteilten Entscheidungsgewalt (Arnstein 1969; Friedman u. Miles 2006). In einer heute zunehmend partizipativen Gesellschaft sollte dabei immer kritisch geprüft werden, was mit der Interaktion und Einbindung bezweckt wird. „Feigenblatt“-Maßnahmen sollten unterlassen werden.

\section{Monitoring/Evaluierung}

Um die Maßnahmen schnell anpassen zu können, wenn sie nicht erfolgreich sind oder wenn sich äußere Bedingungen ändern, ist es notwendig, die Einstellungen der Stakeholder genau zu beobachten. Wenn sich die Charakteristika der Stakeholder ändern, kann eine Änderung der Interaktionsstrategie oder auch eine Änderung des Projektplans notwendig sein (Bjugn u. Casati 2012).

\subsection{Qualitative Interviews mit zentralen Biobanking-Akteuren an vier Universitätskliniken}

Im Rahmen des Teilprojekts Kommunikation/Public Outreach des German Biobank Node wurden im Juli und August 2014 an vier Universitätskliniken, die zentralisierte Biobanken aufbauen, semistrukturierte Interviews mit zentralen Biobanking-Akteuren geführt. Gesprächspartner waren zum einen die Biobankbetreiber (Führungskräfte und Mitarbeiter der zentralisierten Biobank, $\mathrm{n}=10$ ), zum anderen (Grundlagen-)Wissenschaftler als potenzielle Probennutzer $(n=3)$ sowie forschende Kliniker $(n=6)$, die Proben selbst bei ihren Patienten sammeln, um diese für ihre Forschung zu nutzen. Die Interviews wurden im Zeitraum Februar bis Juli 2015 ausgewertet, vollständig 
transkribiert, von beiden Interviewern (AS, WL) kodiert und schließlich kategorisiert, wobei hierfür sowohl die ursprünglichen Fragestellungen als auch Themen, die sich aus den Interviews ergaben, leitend waren.

Ziel der Analyse war es, Informationen darüber zu gewinnen, welche Voraussetzungen für eine Zusammenarbeit von Wissenschaftlern und Ärzten mit der zentralisierten Biobank erfüllt sein müssen und welche Hemmnisse bestehen. Es sollten die Wünsche und Erwartungen der klinikumsinternen Nutzer an zentralisierte Biobanken erforscht und die Anforderungen an Biobanken-Betreiber hinsichtlich der Kommunikation mit internen wie externen Stakeholdergruppen eruiert werden.

Die verschiedenen Rollen fallen derzeit sehr häufig noch in einer Person zusammen: Diejenigen, die bereits Proben für ihre wissenschaftliche Arbeit nutzen, sind meist auch Kliniker, die ihre Patienten um die Probenspende bitten und oftmals auch selbst Energie in den Aufbau einer entsprechenden krankheitsbezogenen Biobank stecken. Auch diejenigen, die eine zentralisierte Biobank aufbauen und betreiben, sind oft über ihre eigene bisherige Forschungsarbeit zu dieser Aufgabe gekommen. Langfristig wird die Weiterentwicklung von großen Forschungsinfrastrukturen wie Biobanken dazu beitragen, dass humane Proben auch von solchen Wissenschaftlern genutzt werden, die selbst nicht in die Patientenversorgung eingebunden sind, dass Proben im Krankenhaus von Ärzten entnommen werden, die selbst nicht forschen, und dass Biobanken von Personen betrieben werden, für die dies die Hauptaufgabe und ein eigenständiges Berufsbild ist. Bei der Auswertung der Interviews wurden gedankliche Rollenwechsel der Gesprächspartner berücksichtigt.

\subsection{Wissenschaftler: Erwartungen und Wünsche der Probennutzer}

Die Wissenschaftler $(\mathrm{n}=3)$, die in einer reinen Rolle als potenzielle Probennutzer interviewt wurden, waren Grundlagenforscher aus dem Bereich Pharmakologie, die derzeit noch wenig mit humanen Proben forschen, dies aber künftig gerne verstärkt tun würden. Die Arbeitsweise und der Nutzen der zentralisierten Biobank an ihrem Standort waren ihnen noch weitgehend unbekannt. Besonders wichtig ist für sie, dass die Proben hinsichtlich ihrer Qualität gut charakterisiert und dokumentiert sind:

„Das Kollektiv [...] muss ausreichend groß sein, es muss möglichst gut dokumentiert sein, es muss histopathologisch gut dokumentiert sein, [...]wir müssen genau wissen, wo wir uns bewegen, um irgendwie sinnvolle Aussagen machen zu können." (Wissenschaftler)

Neben der Verknüpfung der Proben mit klinischen Daten wird perspektivisch auch eine Anreicherung um Daten aus bereits durchgeführten Analysen ge- 
2 Was erwarten Wissenschaftler von zentralisierten Biobanken?

wünscht. Dazu erscheint eine größere Bereitschaft von Wissenschaftlern, ihre Daten mit anderen zu teilen, notwendig:

“... wo die beteiligten Wissenschaftler offen sein müssen, die damit erhobenen Daten wieder zu deponieren, dass man nicht nochmal alle Charakterisierungen für bestimmte Gene wiederholen muss. Das kostet ja alles Geld und das wäre verschwendet, wenn man's schon einmal gemacht hat. Dass sozusagen diese Proben im Laufe der Zeit wie ein Buch beschrieben werden." (Wissenschaftler)

Oberste Priorität hat die einfache Nutzbarkeit der Biobank als Forschungsressource, wie dieses Zitat exemplarisch zeigt:

„Wenn ich Stunden brauche, um die Information aus einer solchen Datenbank herauszuziehen, dann wird das nicht vernünftig machbar sein. Oder wenn ich Leute auf eine spezielle Schulung schicken muss, um Software zu lernen oder ähnliche Sachen, nur um dann auf diese Daten zugreifen zu können, dann wird es sich nicht jedes Labor leisten können." (Wissenschaftler)

Auch verlässliche Zeitabläufe, die Einhaltung von vereinbarten Fristen und feste Ansprechpartner werden erwartet. Sie versprechen eine deutliche Vereinfachung gegenüber dem aktuellen Status, da die Interaktion mit Klinikern aufgrund ihrer hohen Arbeitsbelastung als organisatorisch schwierig gesehen wird.

Als großer Vorteil einer zentralen Biobank-Infrastruktur wird außerdem gesehen, dass diese die Möglichkeit bieten würde, einen Überblick über das am Standort vorhandene Biomaterial und dessen Qualität zu haben. Diesen Wunsch äußern auch die forschenden Kliniker, die hoffen, auf diese Weise auch eine bessere Übersicht über ihre eigenen Probenbestände zu gewinnen:

„Dass eine Art Sampletracking stattfindet, fände ich gut, also dass ich jederzeit weiß, wieviel meines Biomateriales in welcher Qualität ist noch vorhanden, [...]“" (Kliniker)

Zum Zeitpunkt der Interviews war den befragten Wissenschaftlern allerdings noch unklar, wie der Zugang zu den Proben geregelt werden soll.

Insgesamt wird der Aufbau der zentralisierten Biobank als hilfreich angesehen, da sie dazu beitragen wird, bestimmte Abläufe im Klinikum zu standardisieren und zu einem Routinevorgang werden zu lassen.

\subsection{Forschende Kliniker: Erwartungen und Wünsche der Probensammler}

Die von uns interviewten Kliniker $(n=6)$ waren alle selbst auch Forscher und haben häufig Proben für ihre eigenen Forschungsprojekte gesammelt. Viele 
von ihnen hatten eine Biobank für ihre Fragestellung aufgebaut, diese aber nun in die zentralisierte Biobank ihres Standortes integriert.

Zentrales Thema für diese Cruppe, das für die Akzeptanz und damit langfristig den Erfolg der zentralisierten Biobanken entscheidend sein wird, ist die Frage, wie die Ausgestaltung der Governance-Strukturen erfolgt. Dabei geht es immer wieder darum, ob auf die eigenen Proben auch weiterhin ohne Probleme und bürokratische Hürden schnell zugegriffen werden kann:

“... wenn ich jetzt so an den vorherigen Zustand [...] denke, da waren wir natürlich wesentlich flexibler [...]. Eine Biobank wird immer länger brauchen. Du kriegst einen höheren Standard, eine höhere Qualität, aber es dauert einfach, [...].“ (Kliniker)

Auch die mögliche Weitergabe der eigenen Proben an andere Forscher wird immer wieder thematisiert. In die Sammlung der Proben und Daten sind Ressourcen und oft persönliches Engagement geflossen, eine Weitergabe z.B. an wissenschaftliche Konkurrenten wird sehr kritisch gesehen. Gefordert wird deshalb eine Kontrollmöglichkeit:

„Dass ich als derjenige, der einspeist, gefragt werde, in irgendeiner Form, wenn andere damit arbeiten wollen."(Kliniker)

Die forschenden Kliniker sehen durchaus die Vorteile, die eine zentralisierte Lagerung der Biomaterialien im Klinikum in Bezug auf eine sichere Lagerung und die Gewährleistung der (datenschutz-)rechtlichen Vorgaben bringt. Allerdings steht dieser Aspekt in dieser Gruppe wesentlich weniger im Vordergrund als bei den Biobank-Betreibern. Dagegen sehen sie einen Vorteil bei logistischem Support, den sie von der Biobank erhalten können, beispielsweise beim Transport und bei der Aufbereitung der Proben oder bei ihrer Lagerung und dem Probenmanagement:

„[...] also dass mir diese Arbeitsschritte, die damit verbunden sind, abgenommen werden: aliquotieren, abfüllen, verschicken." (Kliniker)

Auf der Wunschliste steht auch die personelle Unterstützung beispielsweise bei der Dokumentation der Proben, da in den Kliniken hierfür häufig keine Ressourcen zur Verfügung stehen.

Insgesamt beschreiben forschende Kliniker eine Reihe von Anreizen für das Einbringen existierender Sammlungen in eine zentralisierte Biobanken-Infrastruktur:

- Organisation und Qualität

- Auffindbarkeit der Proben, Pool an Patientenmaterial, Logistik

- Professionalisierung, Standardisierung, Qualitätsmanagement

- Gemeinsame Infrastruktur

- Vermeidung von Mehrfachanfragen an Patienten 
2 Was erwarten Wissenschaftler von zentralisierten Biobanken?

- Institutionalisierung und Sichtbarkeit

- Institutionalisierung des Biobankings am Standort

- Professionalisierung durch dieZugehörigkeit zu einer größeren Marke

- Höhere Sichtbarkeit über Dachstruktur

- Vernetzung und wissenschaftlicher Mehrwert

- Vernetzung mit anderen Forschergruppen, Synergien

Zentralisierte Biobanken übernehmen zum einen die Lagerung von Proben für andere Forschungsprojekte, zum anderen aber haben sie vielfach auch die Aufgabe, Restmaterial oder zusätzlich gewonnenes Biomaterial aus dem Behandlungskontext einzulagern und sie - zusammen mit den zugehörigen klinischen Daten - für künftige Analysen zur Verfügung zu stellen. Nicht berücksichtigt wurden bei der Auswahl der Gesprächspartner für die Interviews Ärzte des Klinikums, die selbst keine Forschung betreiben, aber künftig im Behandlungskontext Proben für die Biobank sammeln sollen. 2014 war der Aufbauprozess zentralisierter Biobanken an den meisten deutschen Standorten noch nicht so weit vorangeschritten, dass eine solche Befragung schon sinnvolle Ergebnisse erbracht hätte.

\subsection{Biobankbetreiber: Perspektive der Probenverwalter}

Die Biobankbetreiber - Führungskräfte und Mitarbeiter der zentralisierten Biobanken - $(\mathrm{n}=10)$ standen im Zentrum der Interviews, da es auch darum ging, herauszufinden, wie der „German Biobank Node“ sie hinsichtlich ihrer Kommunikationsaufgaben unterstützen kann. Naturgemäß fällt es ihnen leichter, den Mehrwert einer zentralisierten Biobank an einem Universitätsklinikum zu benennen, als dies bei den Wissenschaftlern und Klinikern der Fall ist, die sich bisher noch wenig mit der neuen Infrastruktureinrichtung beschäftigt haben. Ihre Hauptargumente sind zum einen eine bessere Qualität der Proben und Daten, zum anderen eine höhere Sicherheit sowohl in Bezug auf rechtliche und ethische Anforderungen einschließlich geregelter Governance-Strukturen als auch hinsichtlich einer sicheren Probenlagerung, bei der zentrale Kryolager z.B. mit einer Ausfallsicherung versehen sind oder klare Ausfallkonzepte (Krisenmanagement) bis hin zu Ausweichlagern bestehen bzw. festgelegt sind.

„Die Zustände sind wahrscheinlich an jedem großen Universitätsklinikum erschreckend, und jeden Monat kommt jemand zu mir und sagt, Jetzt will ich doch in die Biobank, denn bei mir ist eine Truhe ausgefallen, die war nicht überwacht, die ist am Wochenende ausgefallen - 15 Jahre Arbeit weg.' Das gibt's wirklich ständig." (Biobankbetreiber)

Auch die Institutionalisierung und damit eine nach außen wahrnehmbare Professionalisierung des Biobanking durch die zentrale Infrastruktur werden hervorgehoben: 
„[...] die Perspektive, dadurch eine fakultätsweite Institution zu schaffen, die dann auch über entsprechende Mittel oder Potenz verfügt, das Biobanking so einzurichten, dass man dann auch sagen kann: , Ich betreibe das institutionell '... Und das kann nur nützlich sein.“(Biobankbetreiber)

Zentrale Biobanken-Infrastrukturen können auch zu einer besseren Übersicht über die in einem Forschungsprojekt genutzten Proben und Daten beitragen und so die Reproduzierbarkeit von Forschung verbessern helfen sowie eine Nachnutzbarkeit von Proben und Daten für zukünftige Projekte ermöglichen. Darüber hinaus können durch eine bessere Sichtbarkeit der Proben oder durch wissenschaftliche Kooperationen, die sich über die Biobank ergeben könnten, Synergien entstehen.

„Aber das heißt eben nicht, dass man nicht durch diese Professionalisierung so eines Netzwerks neue Leute ins Boot bekommt, mit denen man in Zukunft neue wissenschaftliche Projekte anstrengt. [...] man proliferiert sein wissenschaftliches Thema in eine andere Richtung und plötzlich ist man Co-Autor auf vier weiteren Papers, weil man eigentlich nur die Idee weitergetragen hat." (Biobankbetreiber)

Ein weiterer Faktor, der sich positiv vor allem für das Klinikum bzw. die Fakultät auswirkt, ist die Kosteneffizienz, beispielsweise beim Energieverbrauch, wenn nicht mehr jedes Institut und jede Klinik eigene Tiefkühltruhen betreibt.

„[...] Sie können natürlich niemandem verbieten, dass er sich auch in Zukunft hier vollkommen abkapselt. Aber dann wird es keine Unterstützung der Fakultät mehr geben. Und am Ende des Tages müssen sie dann über Gemeinkosten, Strom ... das muss ja alles bezahlt werden." (Biobankbetreiber)

Auch die gemeinsame IT-Infrastruktur und das Datenbank-Management werden als wichtiger Nutzen genannt. Darüber hinaus müssen auch die Anfragen an Patienten nach einer Probenspende und/oder Studienbeteiligung zunehmend besser gebündelt werden, da einer wachsenden Zahl wissenschaftlicher Projekte eine eher abnehmende Bereitschaft zur Teilnahme an klinisch-wissenschaftlichen Studien gegenüber steht.

„Das Problem kommt erst, wenn es zu viele Studien gibt, die Gewebe haben wollen, [...] dass da nicht fünf Forscher hingehen und jeder seinen eigenen Aufklärungsbogen hat und der Patient nachher fünf Mal unterschreiben muss. Das hatten wir früher immer, dass dann die Leute gesagt haben, ich habe schon für drei Sachen unterschrieben, ich will jetzt nicht noch für das Vierte oder Fünfte unterschreiben, egal, ob es jetzt etwas anderes ist..." (Kliniker)

Eine zentralisierte Biobank als neue Infrastruktur in einem Universitätsklinikum muss zunächst vor allem bei den klinischen Kollegen Akzeptanz fin- 
2 Was erwarten Wissenschaftler von zentralisierten Biobanken?

Eine qualitative Stakeholder-Analyse

den. Dies gelingt nach Erfahrung dann leichter, wenn der Kliniker selbst an Forschung interessiert ist:

„Die [...] haben eigentlich nur dann eine echte Motivation, wenn sie ein grundsätzliches wissenschaftliches Interesse haben und meistens dann auch selber mit den Proben und Daten arbeiten, und dann im Gegenzug für das Zur-Verfügung-Stellen auch andere Proben und Daten bekommen, die sie ja brauchen, als Kontrolldaten oder -proben." (Kliniker)

Wiederholt betont wurde auch, dass die Probengewinnungs- und -verarbeitungsprozesse gut in die klinischen Routineabläufe integriert werden müssen.

Alle Biobankbetreiber betonen, wie wichtig Governance-Strukturen sind, um den Forschern, die „ihre“ Proben in der Biobank einlagern, eine gewisse Kontrolle über ihre Nutzung und einfachen Zugriff für ihre eigene Forschung zu gewähren. Zugleich ist der Sinn der zentralisierten Biobank aber doch auch, die häufig mit öffentlichen Mitteln - wenn auch häufig mit viel privater Zeit - gewonnenen Proben und Daten auch für eine breitere Nutzung verfügbar zu machen.

„Ob der einzelne gerne seine Kontrolle abgibt oder ob er das Gefühl hat, dass er sie abgeben muss und das gar nicht so gerne will, das ist ja noch ein ganz anderes Thema im klinischen Bereich. Es geht immer auch um Daten, wo Leute teilweise ihr Herzblut oder ihre Initiative, ihre Energie rein verwenden ... Das ist [...] im akademischen Bereich leider Cottes nun mal so, dass es häufig dazu führt, dass so der Gedanke des privaten Anspruchs an irgendwelche Daten oder auch Proben, die eigentlich Allgemeingut sind, zustande kommt."(Biobankbetreiber)

Als Faktor, der für die Akzeptanz der zentralisierten Biobank förderlich ist, wird auch ihre Neutralität genannt, wenn sie eine Einrichtung des Klinikums oder der Fakultät ist und nicht von einer Klinik oder einem Institut betrieben wird:

„Das ist wesentlich neutraler, es ist eine große Gefahr, dass dann dieses Institut die Proben für sich vereinnahmt und damit publiziert, [...]“ (Biobankbetreiber)

Es gilt also, Vertrauen zu schaffen. Als wichtiger Faktor hierfür haben sich auch das persönliche Engagement und die Intensität der internen Vernetzung der Biobankbetreiber erwiesen. In die persönliche Kontaktaufnahme und Vorstellung der Biobank fließt in der Aufbauphase viel Zeit der Führungskräfte:

„Was wir dann gemacht haben das erste halbe Jahr [...] habe ich wirklich mal mit jedem Arbeitsgruppenleiter, mit jedem Klinikdirektor, jedem Institutsleiter gesprochen, [...].“ (Biobankbetreiber) 
Sehr erleichtert wird der Etablierungsprozess, wenn die zentralisierte Biobank explizit von der Fakultäts- und/oder Klinikumsleitung sowie von der Ethikkommission unterstützt wird:

„Es gibt die klare Ansage der Fakultät, dass die Fakultät das so will und dass Biobankingaktivitäten, wenn sie noch in irgendeiner Weise an Fakulätsressourcen hier partizipieren wollen, sich dem unterzuordnen haben." (Biobankbetreiber)

Nicht zuletzt wird der Faktor Zeit eine große Rolle spielen. Zentralisierte Biobanken sind neu, und sie sind ein Element in einer Entwicklung zu immer größeren und vernetzteren Forschungsinfrastrukturen in der Medizin. Der angestoßene kulturelle Wandel wird sich vollziehen, und die Biobankbetreiber sind davon überzeugt, dass sie zunächst mit denen voranschreiten können, die motiviert sind, und dass der Rest folgen wird.

„Da wird schon immer wieder auf den Stein getropft [...] je mehr die Leute mit uns zusammenarbeiten, je zufriedener die sind und je mehr die das kommunizieren, desto mehr wird das hier auch kippen ...." (Biobankbetreiber)

Befragt nach ihren wichtigsten Kommunikationsaufgaben sagen die Biobankbetreiber übereinstimmend, dass die interne Kommunikation am Standort zunächst vordringlich ist, um die Bekanntheit der Biobank zu steigern und ihren Nutzen zu vermitteln sowie auch, um die Logistik der vielen an dem gesamten Prozess Beteiligten zu planen und umzusetzen.

„[Gebraucht würde] ein generisches Konzept dafür, wie man so eine Kommunikation in so einem Universitätsklinikum [gestaltet] zu den Kliniken, die [...] über Materialien verfügen, aber sich zurückhalten, sich in so ein Netzwerk mit einzubringen, [...]." (Biobankbetreiber)

Es wird auch darauf hingewiesen, dass (zentralisierte) Biobanken zum Zeitpunkt der Interviews selbst bei Klinikern der jeweiligen Standorte noch nicht sehr bekannt waren. Insbesondere solche Kliniker, die selbst nicht forschen, sollten hierzu informiert werden.

„Man muss in die Fachgesellschaften gehen und [...] ein bisschen Trendsetting betreiben. [...] Also die, die sowieso forschen, die braucht man auch glaube ich nicht zu überzeugen. Die, die aber kaum oder sehr eingeschränkt oder gar nicht forschen, das gibt es nun mal auch, auch im Universitätsklinikum, die zu gewinnen [...]." (Biobankbetreiber)

Zur internen Kommunikation gehört auch, den Informationsfluss zur Biobank zu managen. So wäre zu fordern, dass die zentralisierte Biobank einen Überblick darüber hat, welche Sammlungen am Standort vorhanden sind, und dass Ergebnisse und Publikationen zu Forschungsprojekten, die unter 
2 Was erwarten Wissenschaftler von zentralisierten Biobanken?

Eine qualitative Stakeholder-Analyse

Nutzung von Proben aus der zentralisierten Biobank entstanden sind, rückgemeldet werden.

„Das ist mühsam, aber das wollen wir erreichen. [...] viele Kliniker, die richtig normalen klinischen Betrieb nebenher haben, sagen ,Bitte bleib mir fern mit allem, was hier zusätzlich ist." (Biobankbetreiber)

\subsection{Eine Kommunikationsstrategie für klinikinterne Stakeholdergruppen}

Die Interviews mit Wissenschaftlern, Klinikern und Biobankbetreibern in vier Universitätskliniken haben gezeigt, dass derzeit die zentralisierten Biobanken als Forschungsinfrastruktur bei den klinikinternen Stakeholdergruppen noch wenig bekannt sind. Gerade bei denjenigen, die aus eigenem Forschungsinteresse Proben sammeln und die dafür gewonnen werden sollen, ihre Sammlungen in der zentralen Biobank zu lagern, bestehen noch zahlreiche Unklarheiten über den konkreten Nutzen, den sie davon hätten. Es bestehen darüber hinaus auch Ängste und Vorbehalte hinsichtlich der Zugriffsmöglichkeiten auf die eigenen Proben. Zugleich wurden von den von uns befragten Wissenschaftlern Erwartungen und Wünsche an Services der Biobank geäußert, die eventuell nicht erfüllt werden können (z.B. Bereitstellung von Personal für die Dokumentation oder Bereitstellung von Analysen, die bereits mit den Proben durchgeführt worden sind).

Während der Aufbauphase einer zentralisierten Biobank wird die Kommunikationsstrategie zunächst stärker auf die forschenden Kliniker fokussieren. Je mehr Proben und Daten dann verfügbar sind, desto mehr wird es auch darauf ankommen, Wissenschaftler für eine Nutzung der Proben und Daten zu gewinnen und entsprechende Services aufzubauen. Hierfür werden weitere Bedarfserhebungen unerlässlich sein.

Für die zentralisierten Biobanken lassen sich aus der Analyse eine Reihe von Kommunikationsaufgaben ableiten:

- Sie müssen die Bekanntheit ihrer Einrichtung im Klinikum/am Standort erhöhen.

- Sie müssen den Mehrwert und persönlichen Nutzen für den Kliniker und/oder Forscher überzeugend darstellen.

- Um im Klinikum Akzeptanz zu finden, müssen die Biobank-Akteure dafür sorgen, dass sie von der Leitungsebene (Dekan, Klinikumsvorstand) sowie von der Ethikkommission vor Ort unterstützt werden.

- Die Leitungspersonen der Biobank müssen sich persönlich für die Vernetzung im Klinikum engagieren.

- Alle Mitarbeiter müssen durch Verlässlichkeit und klare Kommunikation Vertrauen bei den Partnern (Klinikern und/oder Forschern) vor Ort schaffen. 
- Es ist notwendig, den Forschern und Klinikern gegenüber klar zu kommunizieren, welche Leistungen die Biobank erbringen kann und welche nicht.

- Vor dem Hintergrund einer zunehmend partizipativen Gesellschaft und dem Aufbau größerer und damit „anonymerer“ Forschungsinfrastrukturen darf die Kommunikation mit Probenspendern und die Transparenz gegenüber der Öffentlichkeit nicht vernachlässigt werden - auch wenn dies im ersten Moment noch nicht so sehr dringlich erscheint.

Der German Biobank Node wird die Biobank-Betreiber in Deutschland auf dieser Basis mit verschiedenen übergeordneten Maßnahmen unterstützen. So werden Materialien in verschiedenen Formaten bereitgestellt, die von den Biobanken für ihre Nutzerkommunikation verwendet werden können. Es ist geplant, einen Leitfaden zur Kommunikation zu erstellen, der auch Empfehlungen für die standortinterne Kommunikation der Biobanken enthalten soll. Außerdem werden die Ergebnisse des Projektes in ein Fortbildungsangebot zum Thema Stakeholder Engagement für Biobankbetreiber einfließen.

\section{Tipps für die klinikinterne Kommunikation zentralisierter Biobanken}

- Etablieren Sie Ihre Biobank als Marke innerhalb Ihrer Einrichtung.

- Verstehen Sie die Stakeholder-Kommunikation als Führungsaufgabe. Planen Sie personelle und finanzielle Ressourcen für die Kommunikation ein.

- Nutzen Sie die vorhandenen klinikumsinternen Medien (MitarbeiterZeitschrift, Intranet, „schwarze Bretter" etc.), um die Biobank und ihren Nutzen bekannt zu machen. Alle Mitarbeiter der Klinik sollten regelmäßig über die Biobank „stolpern“. Arbeiten Sie möglichst mit der Pressestelle Ihres Klinikums oder Ihrer Fakultät zusammen.

- Eine Informationskampagne zum Thema Biobanken, die sich an die Patienten richtet, wirkt auch beim Personal.

- Kommunizieren Sie positive öffentliche Darstellungen und Meinungen zum Thema auch nach innen.

- Stellen Sie laienverständliche Informationen und News aus der Forschung über die Website Ihrer Biobank zur Verfügung - auch die Mitarbeiter des Klinikums nehmen kurze und verständliche Informationen besser auf.

- Persönliche Begegnungen stärken das Vertrauen. Veranstalten Sie einen Biobanking-Tag mit Führungen und Vorträgen für die Klinikmitarbeiter. Besuchen Sie Jour fixes oder Frühbesprechungen der Kliniken, um Ihre Biobank vorzustellen.

- Bieten Sie Fortbildungen für die Mitarbeiter des Klinikums an und schaffen Sie positive Anreize für die aktive Beteiligung.

- Beteiligen Sie die Wissenschaftler vor Ort an der Ausgestaltung und Weiterentwicklung der Governance-Strukturen Ihrer Biobank. Ein 
2 Was erwarten Wissenschaftler von zentralisierten Biobanken?

Eine qualitative Stakeholder-Analyse

Round table oder eine vergleichbare Dialogveranstaltung kann helfen, das Feedback und den Bedarf der Probensammler und Probennutzer am Standort einzuholen. Nehmen Sie die geäußerten Bedürfnisse und Bedenken ernst.

- Machen Sie die Meinungsführer in der Klinik ausfindig und versuchen Sie diese für die Sache der Biobank zu gewinnen.

- Pflegen Sie ein gutes Verhältnis zur Fakultäts-und/oder Klinikumsleitung und binden Sie die lokale Ethik-Kommission frühzeitig in den Aufbau und die Ausgestaltung der Biobank ein.

- Bieten Sie Ihren Nutzern Service, beispielsweise gut funktionierende IT-Tools. Gewährleisten Sie einen möglichst geringen administrativen Aufwand bei Probenanfrage.

- Prüfen Sie, wie Sie dazu beitragen können, die Forscher am Standort disziplinübergreifend untereinander zu vernetzen, um so neue Forschungsideen zu generieren. Hierzu könnte die Biobank beispielsweise ein klinikinternes Symposium veranstalten oder einen Think tank für Nachwuchsforscher initiieren.

\section{Quellenangaben}

Arnstein S.R. (1969): A ladder of citizen participation. J. Am. Inst. Plann. 35, 216-224.

Bjugn R., Casati B. (2012): Stakeholder Analysis: A Useful Tool for Biobank Planning. Biopreservation Biobanking 10, 239-244.

Buse K., Mays N., Walt G. (2012): Making Health Policy, Second Edition. ed. Open University Press.

Caixeiro N.J., Byun H.L., Descallar J., Levesque J.V., de Souza P., Soon Lee C. (2015): Health professionals' opinions on supporting a cancer biobank: identification of barriers to combat biobanking pitfalls. Eur. J. Hum. Genet.

Cañada J.A., Tupasela A., Snell K. (2015): Beyond and within public engagement: a broadened approach to engagement in biobanking. New Genet. Soc. 0, 1-22.

European Commission (2012): Biobanks For Europe.

Friedman A.L., Miles S. (2006): Stakeholders. Theory and Practice.

Gaskell G., Gottweis, H., (2011): Biobanks need publicity. Nature 471, 159-160.

Gottweis H., Lauss G. (2012): Biobank governance: heterogeneous modes of ordering and democratization. I Community Genet.

Lesch W., Schütt A., Jahns R. (2016): Biobanken in der öffentlichen Wahrnehmung: Verständnis, Interesse und Motivation von Probenspendern in Deutschland, in: Gesundheitsforschung Kommunizieren, Stakeholder Engagement gestalten. Grundlagen, Praxistipps Und Trends, TMF-Schriftenreihe. MWV, Berlin.

Mitchell D., Geissler J., Parry-Jones A., Keulen H., Schmitt D.C., Vavassori R., Matharoo-Ball B. (2015): Biobanking from the patient perspective. Res. Involv. Engagem. 1, 4

Simeon-Dubach D., Watson P. (2014): Biobanking 3.0: Evidence based and customer focused biobanking. Clin. Biochem. Volume 47, 300-308.

Das Projekt wurde vom Bundesministerium für Bildung und Forschung im Rahmen des German Biobank Node unter dem Förderkennzeichen 01EY1301 gefördert. 